\title{
Enjeux géohistoriques et politiques de la conscription aux Emirats arabes unis
}

\section{Léa Fabre}

\section{OpenEdition}

\section{Journals}

Édition électronique

URL : https://journals.openedition.org/geohist/802

DOI : $10.4000 /$ geohist.802

ISSN : 2264-2617

Éditeur

Association française de la Revue de géographie historique

Référence électronique

Léa Fabre, «Enjeux géohistoriques et politiques de la conscription aux Emirats arabes unis », Revue de géographie historique [En ligne], 12 | 2018, mis en ligne le 20 mai 2018, consulté le 12 juin 2021. URL: http://journals.openedition.org/geohist/802 ; DOI : https://doi.org/10.4000/geohist.802

Ce document a été généré automatiquement le 12 juin 2021.

\section{c)}

Ce(tte) œuvre est mise à disposition selon les termes de la Licence Creative Commons Attribution Pas d'Utilisation Commerciale - Pas de Modification 4.0 International. 


\title{
Enjeux géohistoriques et politiques de la conscription aux Emirats arabes unis
}

\author{
Léa Fabre
}

1 La loi sur le service national, annoncée en janvier 2014 par le vice-président des Émirats Arabes Unis, Ministre de la Défense et émir de Dubaï Mohammed ben Rachid Al Maktoum, est particulièrement intéressante eu égard à plusieurs caractéristiques communes aux États du Golfe et que Victor Gervais résume ainsi : "L'élaboration d’un outil de défense s'est d'abord heurtée aux conditions socioéconomiques et culturelles de ces pays, handicapés à la fois par l'absence de tradition militaire moderne, par le manque de main d'œuvre (qualifiée surtout) et par les difficultés de recrutement dues à l'enrichissement de leur population. Mais c'est davantage le mode particulier de formation de ces États qui rend l'option militaire aussi coûteuse. Il existe en effet parmi les dirigeants du Golfe une réticence évidente à intégrer leur population dans l'effort de défense du pays, par l'appréhension inquiete de voir celles-ci s'immiscer dans le processus de prise de décision, ou, plus significativement, par la crainte longtemps nourrie des putschs militaires anti-monarchiques dans la région. Par ailleurs [...] le pacte social sur lequel s'érige l'État rentier du Golfe se pose précisément en obstacle à la mobilisation de la société pour la défense du pays $»^{1}$.

2 La conscription est une forme de service militaire dans laquelle l'État requiert qu'un pourcentage d'hommes serve dans l'armée sous peine de conséquences légales. Elle permet à l'état de recruter des soldats à moindre frais, comparativement à l'emploi de mercenaires étrangers ou de volontaires pour qui l'engagement dans l'armée doit être suffisamment attrayant pour compenser le risque afférent. L'instauration du service militaire obligatoire permet en outre de disposer d'une force de réserve importante mobilisable en tout temps et dans un temps relativement court. Eu égard aux contraintes attachées à la conscription, à savoir l'impact que la ponction d'hommes peut avoir sur l'économie du pays, elle est généralement utilisée lorsque l'État se sent en insécurité dans son environnement stratégique proche. Au-delà des considérations militaires et sécuritaires, la conscription peut également être utilisée dans un but 
politique, afin d'inculquer certaines valeurs attachées à l'institution militaire - la loyauté, la discipline - et porteuses de sentiments nationalistes. Elle est également l'occasion de réaliser un brassage social d'individus issus de milieux socioculturels différents et donc d'achever la cohésion nationale.

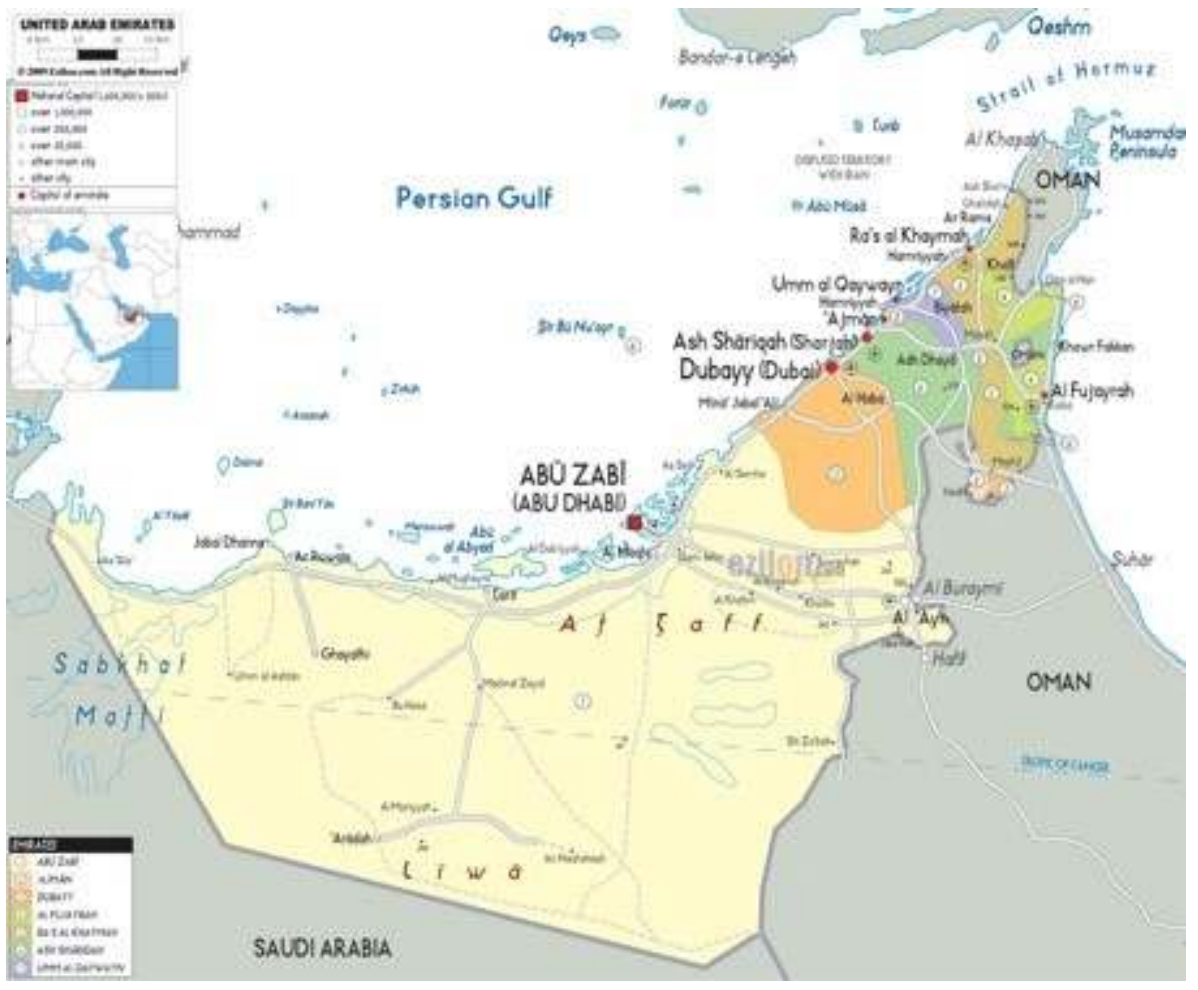

Le projet de création d'un service national et d'une force de réserve aux Emirats Arabes Unis date du début des années 1990 et résultait de la volonté d'Abou Dhabi de faire des forces armées "le ciment de la nation émiratie en formation ${ }^{2}$. En effet, Victor Gervais explique dans sa thèse que " la participation contrainte de la population à l'effort de défense est apparue comme un moyen utile pour assurer à la fois les objectifs de développement et d'" émiratisation" des forces armées. Au niveau politique, l'instauration d'un service militaire obligatoire par les dirigeants émiratis soulignait une volonté de rétablir les forces armées comme lieu de fabrication des "sentiments nationalistes", qui avait fait recette dans les régimes républicains de la région au cours des décennies précédentes »3. Or, les Émirats du Nord, historiquement moins impliqués dans le processus fédéral, se sont opposés à cette proposition. L'idée a cependant continué de germer, et depuis 2011, il n'y a plus aucune résistance possible face à la volonté politique d'Abou Dhabi en raison de la crise économique et de l'affaiblissement des autres Emirats. Dès lors, la loi a pu être discutée et mise en place avec une rapidité mettant en lumière une impulsion présidentielle claire et une volonté politique forte.

4 Cette loi sur le service national marque un tournant dans la vie politique des EAU. Elle n'est qu'une étape dans le processus en cours dans le pays pour faire des forces armées le cœur de la construction étatique. La fin prochaine du pétrole - environ un siècle d'exploitation au rythme actuel -, les problématiques démographiques ${ }^{4}$ dont fait partie l'occidentalisation des comportements, ou encore le chômage des jeunes sont autant de défis qui peuvent mettre à mal l'unité de la Fédération et sa prospérité économique et politique. Dans ces conditions, c'est par le service national que les dirigeants émiriens ont cherché à souder la population autour de valeurs patriotiques. 


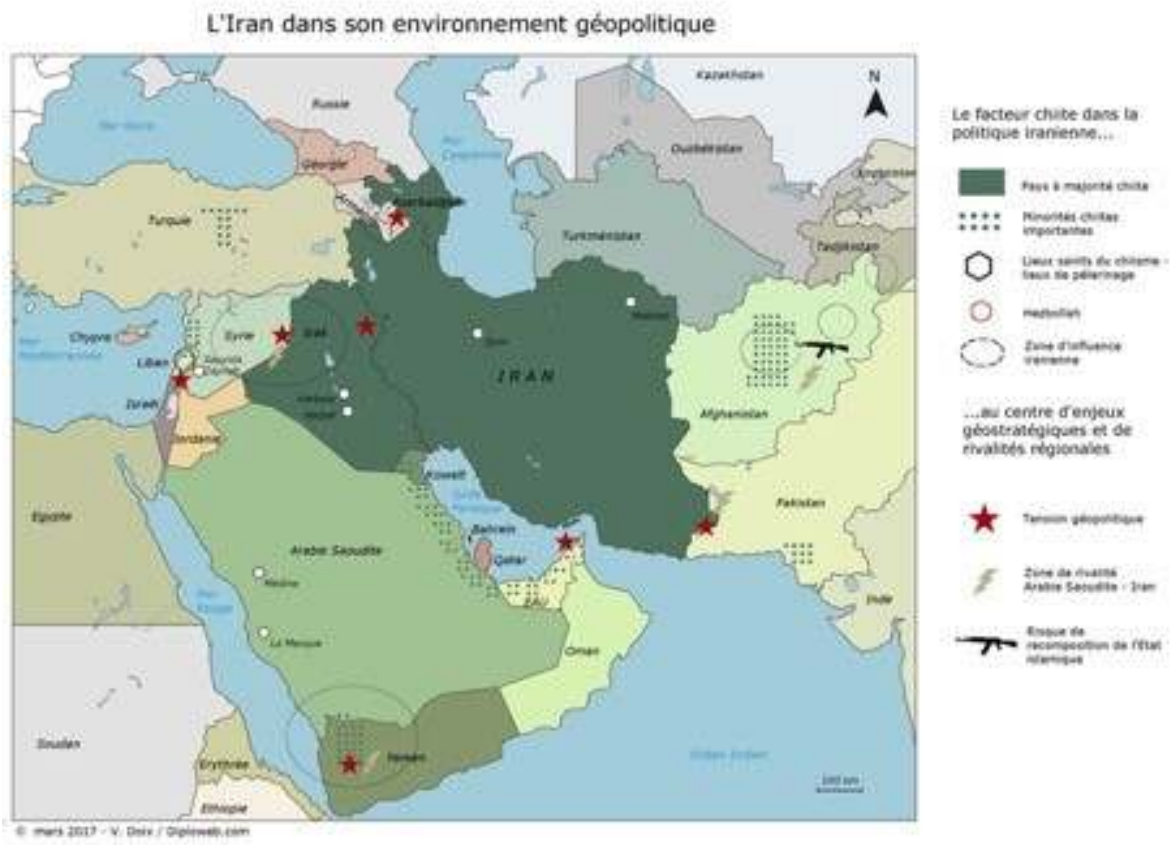

5 Ce changement de paradigme et la place centrale qu'occupent désormais les forces armées dans l'édification du roman national invitent à s'interroger sur les ressorts de la décision d'instaurer un service national : que nous apprend cette loi sur la situation géopolitique des Emirats Arabes Unis? La volonté de dépassement des fragilités internes va de pair avec l'affirmation d'une politique étrangère originale dans le Golfe qui requiert la participation accrue des citoyens à son exécution. Le service militaire s'inscrit à l'intersection de ces deux dimensions, et permet, par la mobilisation d'un récit national, de révéler une nouvelle ambition régionale.

\section{L'émergence progressive d'une conscience nationale...}

6 La loi sur le service national aux Emirats Arabes Unis impose aux jeunes hommes et permet aux jeunes femmes de 18 à 30 ans d'effectuer un service militaire de douze mois à deux ans. Celui-ci vise à leur inculquer des valeurs de discipline et de loyauté et il est une des manifestations de la volonté des leaders émiriens de faire de l'armée le cœur de la construction nationale.

\section{A. Le contenu de la loi sur le service national}

7 L'objectif principal du service national est le renforcement du sentiment national. En effet, Al Maktoum, émir de Dubaï, présente le service national nouvellement créé comme un devoir sacré devant enraciner chez les jeunes émiratis les valeurs d'allégeance, de loyauté, de discipline et de sacrifice, dans le but affiché d'assurer l'indépendance et la souveraineté du pays et préserver les acquis de la Fédération. En réalité, il s'agit également de pallier au manque chronique d'effectifs dont souffrent les forces armées émiraties dû au déficit d'attractivité de l'armée auprès de la jeunesse. Le Ministère de la Défense a du mal à recruter suffisamment pour satisfaire aux besoins 
qualitatifs et quantitatifs des forces armées, d'autant plus importants en période d'engagement militaire à l'étranger. Pour les dirigeants émiratis, le service national est un moyen de pallier aux carences de l'appareil militaire tout en étant un canal de recrutement si des vocations naissent parmi les recrues.

Le projet de loi est discuté en février au sein du Conseil national Fédéral, il est amendé et entériné le 26 mars 2014, et promulgué le 10 juin. Dans sa version initiale, la loi impose aux hommes âgés de 18 à 30 ans d'effectuer un service militaire dont la durée dépend de leur niveau d'éducation : neuf mois pour ceux ayant le baccalauréat - cette durée est allongée à douze mois en mars 2016 -, et deux ans pour les hommes ayant un niveau scolaire inférieur. Sont exemptés les jeunes hommes étant les responsables financiers et légaux de leur famille, ceux atteints de troubles mentaux ou physiques, les fils uniques, ainsi que ceux ayant déjà effectué un service militaire de plus d'un $\mathrm{an}^{5} \mathrm{Ce}$ service militaire est également accessible aux femmes, sans pour autant être obligatoire. La féminisation des forces armées par la conscription, en plus de renforcer l'image de modernité qu'ont les EAU, permet d'augmenter les effectifs ; elle peut également être vue par les femmes comme un moyen de s'émanciper dans une société qui reste traditionnelle malgré des changements au fil des années. A partir de mars 2016, une nouvelle mesure prévoit pour les personnes âgées de 30 à 40 ans la possibilité d'effectuer quinze semaines d'instruction sur autorisation de leur employeur. Celle-ci aurait été décidée à la suite de réclamations de jeunes gens souhaitant participer à l'effort de défense du pays et dont l'âge ne correspondait pas aux critères d'éligibilité de la loi initiale. Cette information est cependant difficile à confirmer, les données étant inaccessibles et les sources rares.

Dès 2011, le projet de création d'un service national et d'une force de réserve est confié à un Général Finlandais, Markku Koli, qui rend ses recommandations auprès des autorités émiraties en 2013. Celui-ci se serait inspiré des modèles de service national mis en place en Finlande, à Singapour et en Corée du sud. Parallèlement au processus législatif, les dirigeants émiratis décident la mise en place de la NSRA, la National Service and Reserve Authority, dirigée par le Général de Division Aérienne Cheikh Ahmed bin Tahnoun bin Mohammed al Nahyan, appartenant au clan de la famille régnante d'Abou Dabi. Cette nomination laisse penser que la famille al Nahyan tient à verrouiller et à garder le contrôle sur les décisions relatives au service national. La NSRA chapeaute l'action des ministères impliqués dans le service national, et est directement affiliée à l'Etat-Major émirati.

La formation est découpée en trois phases : la première consiste en une formation initiale de trois mois, suivie d'une formation spécialisée de trois mois au sein du Ministère de la Défense, du Ministère de l'Intérieur, des forces armées, des forces de sécurité intérieure ou de la police, et enfin une période de trois à quinze mois dans une unité des forces armées, de la police ou des agences gouvernementales. Toutes les recrues reçoivent le même entrainement, mais la formation varie en fonction de leurs spécialités universitaires ou professionnelles. La formation initiale a lieu dans l'un des cinq camps d'entrainement que compte le pays, l'un d'entre eux étant réservé aux recrues féminines. Une fois le service effectué, les hommes et les femmes rejoignent la réserve nationale, constituée d'anciens membres des forces armées, des conscrits et de volontaires, et peuvent être appelés sous les drapeaux jusqu'à 60 ans pour les officiers et 58 ans pour les autres. 
11 Les autorités mettent en place un arsenal législatif répressif pour punir ceux qui essaieraient de se soustraire au devoir national. Les déserteurs risquent jusqu'à 10 ans de prison en cas de refus de répondre à l'appel à la mobilisation; ceux qui veulent échapper au service militaire risquent quant à eux jusqu'à un an de prison et de 10000 à 20000 euros d'amende. De plus, la loi prévoit que les émiriens ayant accompli leur service national seront prioritaires en matière de recrutement et de promotion ; les employeurs sont par ailleurs contraints de garder les postes de ceux qui partent accomplir leurs obligations militaires et de continuer à leur verser la moitié de leur salaire, l'autre moitié étant versée par l'État. Enfin, un amendement voté en août 2015 autorise les émiriens nés de père étranger à effectuer leur service national. La mise en œuvre effective du service national a lieu les 13 et 17 juillet 2014 dans des centres de recrutement à Abou Dhabi, Al Ain, Sharjah et Al Gharbia. La première incorporation de 2000 recrues a lieu fin août 2014. Lors de la première année d'existence du service national, quatre contingents sont incorporés afin de faire passer en priorité les classes d'âge atteignant les 30 ans. Le deuxième contingent de 1500 recrues est appelé en janvier 2015 ; le troisième en mars 2015, exclusivement composé de volontaires ; les quatrième et cinquième en août 2015 et janvier 2016 ; et le sixième en août 2016. En deux ans d'existence, six contingents ont donc été incorporés. A raison d'environ 2000 recrues par incorporation, cela fait 12000 jeunes formés à la chose militaire en deux ans. En janvier 2017, c'est le 7ème contingent qui commence la formation initiale. Ce rythme très soutenu se ralentit : si quatre incorporations ont lieu la première année, il n'y en aura que deux de 2015 à 2016 et ce chiffre semble se stabiliser aujourd'hui. On peut analyser ce ralentissement comme étant la conséquence de difficultés d'absorption par l'encadrement militaire d'un flux trop important de conscrits.

Dès l'annonce du projet de loi sur le service national, celui-ci bénéficie d'une couverture médiatique très large dans la presse locale. Les médias mettent en avant les bienfaits qu'aura le service national sur la formation morale des jeunes - dont $8 \%$ sont au chômage - et sur leur avenir professionnel, sachant que $25 \%$ des émiriens quittent l'école au cours du secondaire. La presse se fait également l'écho de l'enthousiasme des jeunes à rejoindre les camps d'entrainement et relaye également la présence parmi les recrues de fils d'émirs fiers d'accomplir leur devoir. C'est par exemple le cas des deux fils de l'émir de Dubaï, Cheikh Ahmed et Cheikh Mansoor, ce dernier étant mis en scène en tenue militaire au Yémen. La présence des plus hautes autorités émiraties aux côtés des recrues, notamment lors des cérémonies de remise de diplôme des appelés, montrent leur implication et servent aussi à exalter un sentiment patriotique en faisant comprendre aux recrues que l'État est fier de les voir accomplir leur devoir. Cette symbolique est très forte et met encore une fois en avant le volontarisme politique des autorités sur ce sujet.

14 Malgré des campagnes médiatiques visant à attiser la ferveur de la population émiratie, certaines sources affirment que les conscrits sont frustrés, notamment ceux affectés dans des unités de soutien où les tâches et l'encadrement sont quasi-inexistants. Les missions confiées seraient peu valorisantes et le commandement trop strict, ce qui aurait pour effet de mettre à mal l'un des objectifs principaux du service national, à savoir servir de canal de recrutement pour les forces armées. Le manque d'appétence pour la chose militaire s'explique par les conditions de travail beaucoup plus avantageuses dans le privé ou dans le public où les salaires sont plus élevés. Les jeunes 
sont également rebutés par un engagement opérationnel au Yémen. Les autorités en réaction multiplient les encouragements en direction des conscrits ; le prince hériter Mohammed Ben Zayed déclare en effet en novembre 2016 que ceux-ci sont les " fiertés de la Nation » et que l'État et ses citoyens voient en eux «leur espoir et leur avenir » ${ }^{6}$.

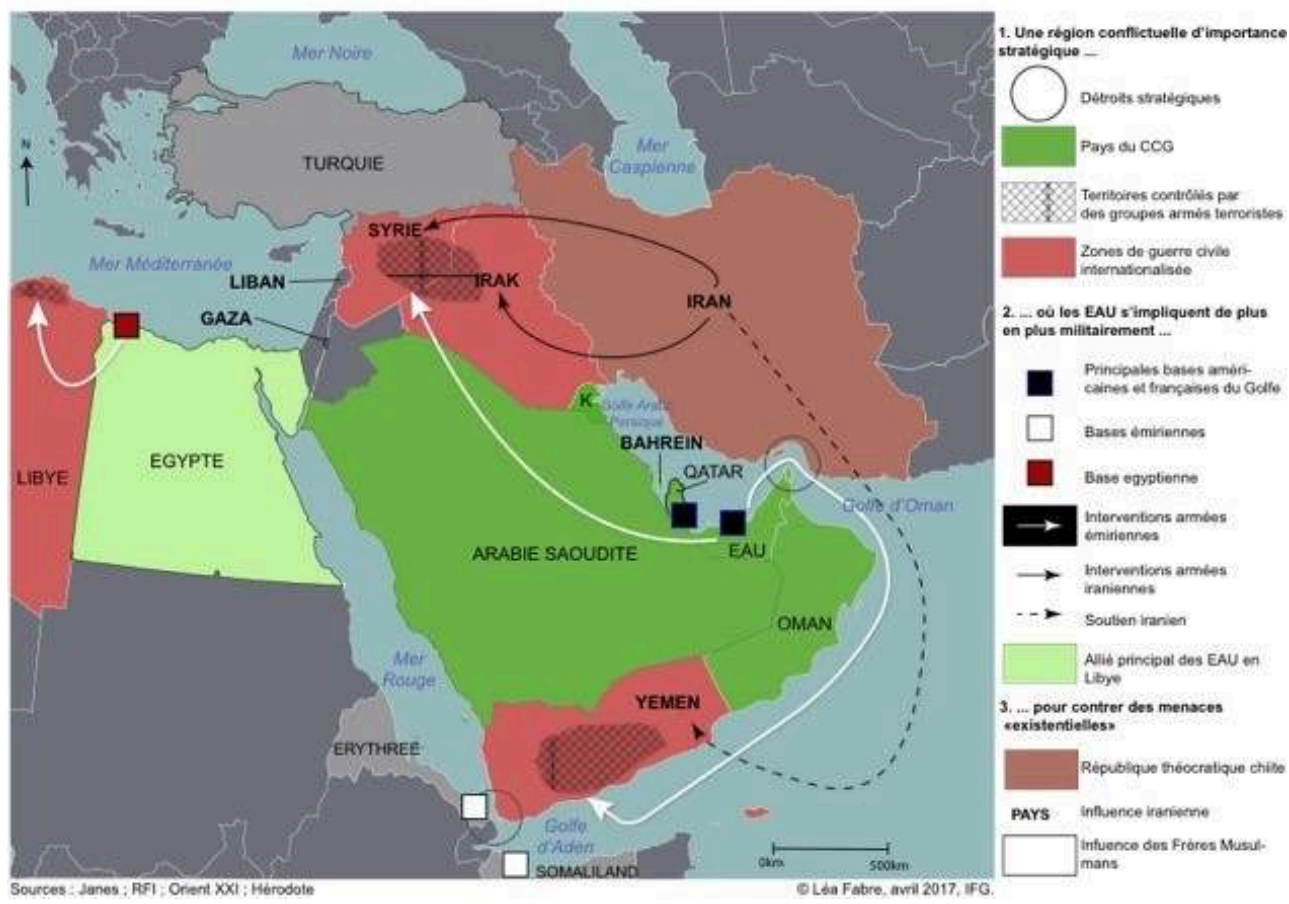

Au-delà de la volonté d'inculquer à la jeunesse des valeurs de discipline et de loyauté, la mise en place du service national aux EAU répond au souhait des dirigeants émiratis de voir naître dans le pays un sentiment patriotique à même de souder la population. L'armée est en quelque sorte un outil de structuration de la société. Tous les moyens sont utilisés pour faire naitre dans le pays un lien Armées-Nation.

\section{B. La création d'un héritage et d'une histoire militaire pour renforcer l'identité nationale}

La mise en avant des forces armées et des symboles nationaux tels que l'hymne ou le drapeau a pour objectif de favoriser un sentiment d'adhésion et de fierté d'être émirien et de fierté que les forces émiraties soient engagées à l'extérieur des frontières nationales ; elle sert également à transmettre à la population l'amour de la patrie et à faire émerger une conscience nationale, qui, in fine, permet de faire accepter la guerre et le sacrifice qu'elle requiert. L'émergence d'une conscience nationale se fait au travers de journées où la population locale est invitée à se réunir aux côtés de ses dirigeants autour de célébrations mettant en avant leur identité et leur histoire.

Tous les pans de la société sont intégrés au processus de consolidation du sentiment national. Des campagnes sont lancées sur les réseaux sociaux, mettant en avant la conscience nationale et l'appartenance à la Nation afin de fédérer les émiriens et notamment empêcher la diffusion d'idéologies subversives. La mobilisation des forces armées fait partie intégrante de la stratégie discursive, elles sont mises en scène aussi bien à la télévision dans des clips patriotiques, que dans les rues du pays lors de défilés militaires. Une série documentaire a par ailleurs été commandée par la National Service 
and Reserve Authority : celle-ci suit en quatre épisodes le parcours de jeunes conscrits durant leur formation et est diffusée à partir de juin 2015 sur National Geographic Abu Dhabi.

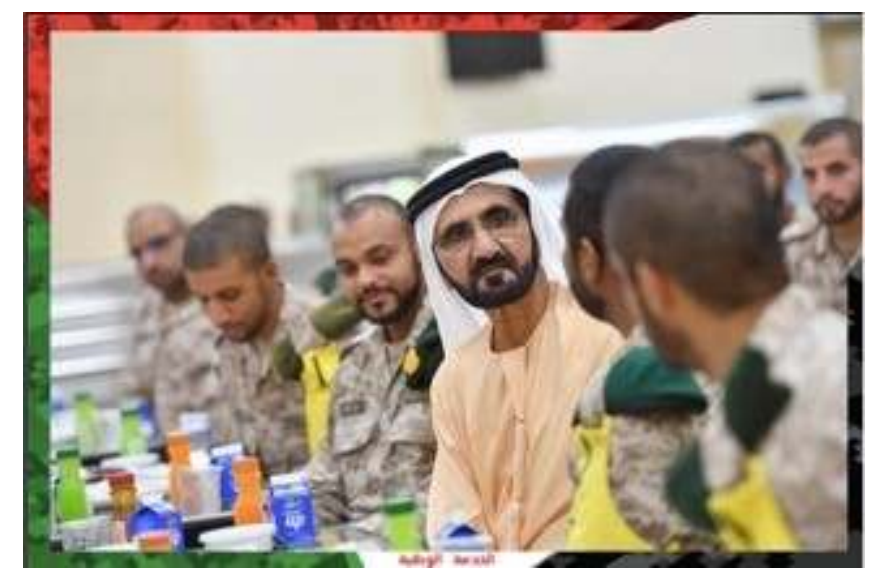

Ces démonstrations martiales visent à galvaniser la population et à introduire au sein de la société la notion de sacrifice pour la Patrie qui lui était jusqu'à présent étrangère, et qui répond à l'engagement de plus en plus lourd des forces armées en dehors des frontières nationales. En effet, bien que les EAU aient déjà subit des pertes humaines lors de précédentes opérations extérieures, celles-ci prennent une toute autre dimension et dans un nouvel ordre de grandeur depuis l'engagement au Yémen en 2015. De lourdes pertes ont succédé à des morts épisodiques. Il était dès lors capital pour les autorités émiriennes de faire accepter à la population locale ce " sacrifice " nécessaire à la protection des intérêts nationaux. D'autre part, la construction d'une identité et d'une conscience nationales, chère au pouvoir émirien, se fait également à travers l'histoire militaire d'un pays et les figures qui ont donné leur vie pour le défendre.

Il est par ailleurs intéressant de noter que l'utilisation du terme " martyr » pour désigner les soldats émiratis morts en opération emporte avec elle une représentation religieuse et quasi-mystique. En effet, par définition un martyr est une «personne qui a souffert la mort pour sa foi religieuse, pour une cause à laquelle elle se sacrifie ». L'usage de ce terme n'est pas anodin, comme ne l'est pas non plus celui de "sacrifice » régulièrement utilisé dans la presse et par les autorités émiraties : "la mort sacrée, les valeurs sacrificielles, renvoient d'abord et surtout à la figure du martyr. D'origine essentiellement religieuse, cette figure intéresse le politique au plus haut point, lequel va s'efforcer d'en faire un opérateur symbolique efficace $»^{7}$. La mort des émiriens pour leur pays étant sacrée, elle a une portée symbolique et mémorielle très forte.

Ainsi, les morts et blessés et leurs familles sont l'objet de nombreux hommages et autres initiatives. Bien que les dates symboliques existent déjà depuis longtemps - la fête nationale les 2 et 3 décembre, la journée du Drapeau depuis 2007 - elles sont, depuis l'engagement au Yémen en 2015, l'occasion de célébrer les « héros morts pour la Nation » et les martyrs tombés en opération. Ceux-ci bénéficient désormais en plus d'une journée hommage qui leur est consacrée le 30 novembre, depuis 2015. L'année suivante en 2016, cette journée correspond également à l'inauguration du monument aux morts "Wahat Al Karama ", ce qui donne à cette journée et à cette inauguration une charge symbolique forte, la création de lieux de mémoire étant une étape indispensable du processus d'édification d'une histoire militaire et d'une conscience 
nationale. Cet "Oasis de la Dignité » est érigé à la mémoire des soldats, policiers, diplomates et civils qui ont sacrifié leur vie pour leur pays depuis sa fondation en 1971. Du 30 novembre au 3 décembre, les jours sont fériés et donc uniquement consacrés au recueillement et au devoir de mémoire.

21 L'hommage aux soldats est au cœur d'autres initiatives. Par exemple en septembre 2015 est ouvert le Martyrs Family Affairs Office, administration chargée de veiller à ce que les familles de soldats morts à la guerre reçoivent tout ce dont elles ont besoin. Les gestes financiers en faveur des familles, par exemple l'annulation de leurs dettes, le don de propriétés ou l'exemption des frais de scolarité pour les enfants, visent autant à éviter la contestation qu'à attester de la place à part qu'ont ces familles dans la société émirienne. Les martyrs sont également mis à l'honneur dans une série de timbres mise en circulation en novembre 2015 par la poste émirienne et par une pièce de monnaie en argent distribuée par la Banque Centrale des EAU.

Ces mesures viennent en appui d'un engagement proactif du pays sur la scène régionale.

\section{II. ... pour soutenir une nouvelle ambition régionale}

L'action des Émirats Arabes Unis à l'extérieur et à l'intérieur de ses frontières nationales est conditionnée par la perception de certaines menaces et s'adapte désormais aux bouleversements stratégiques induits par les Printemps Arabes en 2011. La politique étrangère du pays se veut plus proactive que simplement défensive comme elle le fut par le passé, c'est en cela qu'elle requiert l'assentiment de la population.

\section{A. L'adaptation à une reconfiguration de l'environnement stratégique depuis 2011}

Les Printemps Arabes, qui ont commencé le 17 décembre 2010 en Tunisie, ont rabattu les cartes régionales au Moyen-Orient en transformant les dynamiques géostratégiques et en faisant émerger de nouvelles considérations sécuritaires pour les acteurs de la région. La chute ou la déstabilisation de régimes forts comme ceux de la Libye, de la Tunisie, de l'Égypte, ou de la Syrie ont entrainé la dissémination du chaos et avec elle la crainte d'un embrasement généralisé par l'effet « domino ».

Les monarchies du Golfe craignent alors tout autant pour leur sécurité nationale que pour l'avenir des régimes en place. Les menaces auxquelles elles sont confrontées sont subnationales et transnationales. En effet, au niveau interne, l'élan démocratique suscité par les Printemps Arabes ainsi que l'arrivée au pouvoir des islamistes en Égypte ou en Tunisie constituent une menace politique contre laquelle les familles régnantes du Golfe ont tenté de se prémunir avec la distribution d'aides financières, des réformes politiques minimales, et l'arrestation d'opposants. Celles-ci se sont crues relativement épargnées, les conditions socio-économiques, terreau de la contestation, divergeant grandement entre les pays d'Afrique du Nord et ceux de la péninsule Arabique. Néanmoins, le soulèvement à Bahreïn en février 2011 fait prendre conscience aux monarchies du Golfe que la contagion peut avoir lieu, d'autant plus que l'Arabie Saoudite et les EAU accusent l'Iran d'instrumentaliser à son profit l'instabilité régionale. La République islamique constitue donc une menace transnationale, accusée 
de vouloir retourner les minorités chiites du Moyen-Orient contre les gouvernements des États sunnites. Si la sécurité a toujours été au cœur des préoccupations des dirigeants émiriens, le renforcement de cette tendance est attribué pour de nombreux observateurs au prince héritier Mohammed Ben Zayed, dont le demi-frère Khalifa Ben Zayed est toujours officiellement président de la Fédération, malgré un AVC en 2014 et ses rares apparitions publiques depuis. La politique étrangère des EAU se restructure donc autour de deux menaces principales, que l'on peut qualifier d'existentielles.

En premier lieu, l'Iran, avec qui le contentieux originel repose sur des disputes territoriales au sujet des îles Tomb et Abu Musa situées dans le Golfe Persique, et occupées par l'Iran respectivement depuis 1971 et $1987^{8}$. Bien que leur superficie soit réduite et que leur intérêt paraisse relatif, leur possession est en réalité stratégique pour le contrôle du détroit d'Ormuz, par lequel transitent 17 millions de barils de pétrole par jour, soit $35 \%$ des échanges maritimes de pétrole et $20 \%$ de son commerce mondial. Au-delà du contentieux territorial, l'influence grandissante de la puissance chiite, vue comme un modèle politique et religieux alternatif et potentiel hégémon régional, constitue un frein aux ambitions des puissances sunnites. Du point de vue interne, la théorie expansionniste iranienne requérant aux chiites du monde entier de prêter allégeance au Guide Suprême de la révolution islamique, les autorités émiriennes craignent que la communauté chiite émirienne - estimée à $15 \%$ - se retourne contre l'État.

En second lieu, c'est l'islam politique qui constitue la deuxième menace qui définit et façonne la politique intérieure et extérieure des Émirats Arabes Unis. En particulier, l'organisation politique et religieuse des Frères Musulmans, dont la stratégie est d'instaurer dans le monde arabe des républiques islamiques élues démocratiquement. Si par nature, les monarchies du Golfe redoutent tout pluralisme politique, leurs relations avec la Confrérie sont complexes et ont pu changer au cours de l'histoire. C'est en 2011 que la position du gouvernement émirien se durcit. L'accession au pouvoir des Frères Musulmans en Égypte après la révolution fait craindre aux monarchies une contagion et une déstabilisation des régimes en place. Ce sont par ailleurs des personnalités fréristes qui réclament à ce moment-là l'ouverture politique en Arabie Saoudite et aux Émirats. Le pouvoir se lance dans une répression féroce au plan intérieur, et la lutte contre l'islam politique et le salafisme au niveau régional devient un axe central de la politique étrangère de la Fédération. Les Émirats affichent en effet clairement leur opposition « à toute forme d'islam susceptible de concourir au développement du djihadisme $»^{9}$, et les Frères ne sont pour eux qu'une émanation parmi d'autres de ce danger. Bien qu'il soit difficile d'évaluer la capacité d'une institution religieuse à transcender les structures tribales existant aux EAU, le péril intérieur est tel qu'il justifie des moyens de surveillance avancés et l'élaboration d'un contre-discours visant à faire du nationalisme, notamment via le service national, un rempart à l'islamisme et au djihadisme. La crainte de l'attentat est particulièrement ancrée dans les esprits : forte de sa modernité et de son multiculturalisme revendiqués, la Fédération est pourtant une cible privilégiée pour les terroristes qui voit en elle la paganisation du monde arabe ${ }^{10}$.

Ces menaces conditionnent la mise en œuvre d'une politique étrangère singulière : les EAU soutiennent les groupes nationalistes en Libye, en Syrie ou au Yémen, tout à la fois contre les groupes islamistes et contre les groupes appuyés par l'Iran, dont le dessein serait de constituer un arc chiite contre l'arc sunnite. Dans cette configuration et au 
regard des divergences politiques existant avec le néanmoins grand allié saoudien, l'existence des EAU est potentiellement menacée de toutes parts. Et ce d'autant plus que la Fédération ne dispose pas d'une profondeur stratégique suffisante pour se contenter d'une posture uniquement défensive. Ceci explique la nécessaire projection de puissance, qui, couplée à l'élaboration d'un contre-discours nationaliste, a pour objectif de garantir la sécurité des EAU et de poser l'État en acteur incontournable au Moyen-Orient. jouer un rôle nouveau à l'échelle régionale. Ce changement de paradigme peut s'interpréter comme un " tournant interventionniste » qui est commun à tous les pays du Golfe. Ceux-ci perçoivent en fait qu'un potentiel désengagement américain fait peser sur eux l'avenir de la sécurité de la région. Ce désengagement, illustré par le " pivot vers l'Asie » souhaité par Obama, est visible depuis quelques années au Moyen-Orient, où les États-Unis ont préféré jouer un rôle minime dans les cessations des violences en Syrie, dans la résolution de la crise des réfugiés en Europe, ou dans la stabilisation de l'Égypte, de la Tunisie ou de la Libye. Dès lors, que ce soit en Syrie ou au Yémen, les États du Golfe ont pris conscience que sans leur capacité à représenter une réelle alternative militaire susceptible de dissuader un État rival ou des groupes armés subversifs, le vide créé par le retrait américain entrainerait la déstabilisation de la région et partant, la possibilité pour l'Iran de prendre le leadership régional, ce que souhaite par-dessus tout empêcher l'Arabie Saoudite, par ailleurs confortée dans sa position par le président Trump ${ }^{12}$. Ce tournant interventionniste tranche donc avec une tradition de diplomatie conciliante et d'arbitrage promue par le fondateur de la Fédération, Cheikh Zayed.

L'image guerrière des EAU, véhiculée par le surnom de "Petite Sparte du Moyen-Orient " employée pour la première en 2014 par le Général Mattis dans un article du Washington Post $^{13}$, tranche néanmoins avec l'état des lieux objectif des forces armées. Ainsi, bien que le budget de la défense soit en constante augmentation depuis 1997, la faiblesse de l'effectif - entre 40 et 50000 personnels - et l'absence de réelle culture militaire empêche les EAU d'accéder au rang de puissance militaire dotée d'une autonomie stratégique effective. On observe toutefois que l'armée émirienne est considérée comme une des plus organisées et efficaces du Golfe, et les récentes mesures prises par le pouvoir visent à dépasser les faiblesses structurelles et à combler le retard accumulé depuis plusieurs années. A cet égard, la projection de puissance illustrée par

Revue de géographie historique, 12 | 2018 
l'ouverture de bases militaires dans la Corne de l'Afrique ${ }^{14}$ et par l'engagement au sol au Yémen marquent un vrai tournant dans l'histoire militaire du pays.

Si l'expérience de la guerre s'est progressivement acquise notamment avec l'intervention militaire en Afghanistan en 2003, c'est avec l'engagement au Yémen que celle-ci s'ancre durablement au sein de la société. Au-delà des buts de guerre affichés par la coalition, la participation des EAU a également pour objectif d'exposer le savoirfaire des troupes et de justifier l'explosion des budgets de défense depuis plusieurs années. Les forces armées ont permis des victoires stratégiques dans ce conflit, notamment le débarquement à Aden en juillet 2015 qui constitue une prouesse technique indéniable. Le pouvoir émirien est convaincu de son succès dans sa lutte sur le terrain contre Al-Qaida et contre les milices Houthistes soutenues par l'Iran, et il considère être le seul à participer efficacement au processus de reconstruction étatique. Le prince héritier d'Abou Dabi Mohammed ben Zayed souhaite maintenir l'effort de guerre autant pour contrer l'influence de l'Iran que pour peser sur le leadership saoudien afin de le faire renoncer à son soutien à la branche locale des Frères Musulmans Al-Islah.

Cela étant, les divergences stratégiques ${ }^{15}$, le manque d'expérience des troupes ainsi que les difficultés afférentes à la guerre asymétrique entrainent l'enlisement du conflit. De plus, la guerre médiatique est d'ores et déjà perdue eu égard à la situation humanitaire dramatique et aux pertes sans précédent essuyées par la Fédération - plus de 120 morts depuis le début de l'engagement, et des centaines de blessés et de syndromes posttraumatiques. On comprend dans cette optique à quel point il est fondamental pour le pouvoir émirien d'obtenir le consentement de la Nation au sacrifice que requiert la guerre, d'autant plus que l'objectif principal du pouvoir émirien est de consolider sa présence dans le sud de la péninsule arabique et dans la Mer Rouge.

$\mathrm{Au}$ final, la mise en œuvre du service national aux Emirats Arabes Unis révèle la nouvelle ambition du leader de la Fédération Mohammed Ben Zayed. Les EAU cherchent à la fois à assurer leur sécurité et leur survie, et par là même à devenir un incontournable acteur régional par la projection de sa puissance. Le recours à l'armée, à ses symboles et à ses valeurs vise à souder la population autour de ses leaders et à faire accepter à la société le sacrifice que requiert le statut auquel la Fédération aspire.

\section{NOTES}

1. GERVAIS Victor, Du pétrole à l'armée : les stratégies de construction de l'État aux EAU, études de l'IRSEM 2011, pp 57-58

2. Ibid., p. 269

3. Id.

4. Faible population locale : moins de 10 millions d'habitants dont $90 \%$ d'étrangers.

5. «A time to serve : fundamental provisions of UAE federal Law No. 6 of 2014 concerning national and reserve service ", http://www.tamimi.com/en/magazine/law-update/section-8/ 
july-august-3/a-time-to-serve.html ; texte de loi en arabe : UAE Federal Law No.6 of 2014 : http:// www.ilo.org/dyn/natlex/natlex4.detail?p_lang=en\&p_isn=98631

6. Wam, « CP conveys message of 'pride' to conscripts”, Khaleej Times, 28 novembre 2016, http:// www.khaleejtimes.com/cp-conveys-message-of-pride-to-conscripts

7. MAALOUF Tina, «Le Martyr : du religieux au politique », Sens Public, 14 février 2005, http:// www.sens-public.org/article120.html

8. BOULANGER Philippe, « Les défis géopolitiques d'une nouvelle puissance régionale : les Émirats arabes unis », Hérodote 2009/2 (n 133), p. 58-91

9. CHER-LEPARRAIN Marc, «Les EAU chasseurs de Frères Musulmans », 16 février 2017, Orient XXI, http://orientxxi.info/magazine/les-emirats-arabes-unis-chasseurs-de-freres-musulmans, 1718

10. Les EAU ont déjà été confrontés au risque terroriste : démantèlement de cellule terroriste en décembre 2012 et août 2015, incidents isolés (institutrice américaine poignardée dans un centre commercial à Abou Dabi en décembre 2014, jogger américain écrasé à Dubaï). Voir les articles suivants : Staff, «Gang of 41 face trial in UAE over ISIL terror plot", The National, 2 août 2015, https://www.thenational.ae/uae/gang-of-41-face-trial-in-uae-over-isil-terror-plot-1.22151 ;

ALTAHER Nada, "American woman stabbed to death inside Abu Dhabi mall toilet ", Gulf News, 2 décembre 2014, http://gulfnews.com/news/uae/crime/american-woman-stabbed-to-deathinside-abu-dhabi-mall-toilet-1.1421232

11. CORDESMAN Anthony H., SHALALA Robert M., MOHAMED Omar, "The Guf Military Balance Volume III", Center For Strategic and International Studies, p. 190, https://csisprod.s3.amazonaws.com/s3fs-public/legacy_files/files/publication/

140407_Cordesman_GulfMilitaryBalance_VolumeIII_Web.pdf

12. YOUNG Karen E., "The Interventionist turn in Gulf States' Foreign Policies”, Arab Gulf States Institute in Washington, 1er juillet 2016, http://www.agsiw.org/wp-content/uploads/2016/06/ Young_Interventionist_ONLINE.pdf

13. CHANDRASEKARAN Rajiv, "The United States has a quiet, potent ally nicknamed 'Little Sparta' ", The Washington Post, 9 novembre 2014, https://www.washingtonpost.com/world/ national-security/in-the-uae-the-united-states-has-a-quiet-potent-ally-nicknamed-little-sparta/ 2014/11/08/3fc6a50c-643a-11e4-836c-83bc4f26eb67_story.html?utm_term=.475e18fbbf82

14. ARDEMAGNI Eleonora, "The Horn of Africa's Growing Importance to the U.A.E.", Middle East Institute, 25 avril 2017, https://www.mei.edu/content/article/horn-africa-s-growingimportance-uae

15. Les EAU et l'Arabie Saoudite ne s'accordent pas sur le soutien inconditionnel de la puissance saoudienne au Président Hadi. Pour les EAU, seule la famille Saleh est en mesure de créer l'unité politique entre les différentes factions politiques et tribales. A cet égard, les EAU considèrent que l'alliance de l'ancien président Saleh avec les Houthistes prétendument soutenus par l'Iran n'était que de circonstance, et la suite des évènements semble leur donner raison. Le soutien des forces émiriennes va donc aux milices sudistes et aux soutiens de la famille Saleh. Voir PARTRICK Neil, « The UAE's war aims in Yemen », Carnegie Endowment for International Peace, 24 octobre 2017, http://carnegieendowment.org/sada/73524 


\section{RÉSUMÉS}

L'annonce de la mise en place de la conscription aux Emirats Arabes Unis en janvier 2014 marque en tournant dans la vie politique et militaire du pays. En effet, la loi sur le service national illustre la volonté du pouvoir émirien de renforcer la cohésion nationale et de souder la population autour de valeurs patriotiques et d'une histoire militaire singulière. Cette ambition nationale répond à la quête de crédibilité et de puissance d'un État qui doit s'adapter à des bouleversements stratégiques régionaux majeurs et à l'émergence ou au renforcement de menaces qui mettent en péril son existence.

The law on national service announced in January 2014 can be analyzed as a shift in the country's political and military traditions. Indeed, the enforcement of conscription shows a will from Emirian authorities to reinforce national cohesion, foster patriotic feelings, and build a proper military history. This national ambition echoes with UAE' quest for regional power, in a context of growing threats of instability and insecurity.

\section{INDEX}

Mots-clés : Emirats Arabes Unis, service national, cohésion nationale, patriotisme, forces armées, interventions armées

Keywords : United Arab Emirates, national service, national cohesion, patriotism, armed forces, military interventions

\section{AUTEUR}

\section{LÉA FABRE}

diplômée du Master 2 de Géopolitique de l'Institut Français de Géopolitique, sous la direction de Philippe Boulanger (Paris 8). 